\title{
The limiting resolution of ice-sheet elevations derived from pulse-limited satellite altimetry
}

\author{
D.J. WINGHAM \\ Mullard Space Science Laboratory, University College London, Holmbury St. Mary, Dorking, Surrey RH5 6.NT, England
}

\begin{abstract}
The problem of improving the spatial resolution of ice-sheet elevations derived from satellite-altimeter observations is investigated theoretically in two dimensions. An equation for the echo is described that allows the elevation to be determined by linear operations on the echo. An approximate solution of the equation is explored to illustrate the behaviour of the solution. The modifications necessary to consider a regularly sampled solution are described and the effects of echo fluctuations on the reconstructed surface are considered. Numerical examples are described. It is shown that, in general terms, the resolution may be limited by sampling or by echo fluctuations but that in the case of current practical measurements it is the sampling that determines the resolution. This resolution is smaller than has been considered possible hitherto. Limitations of the method are discussed together with extensions to a wider class of problems including three-dimensional reconstructions.
\end{abstract}

\section{LIST OF SYMBOLS}

c Velocity of propagation

$E\{$.$\} Expectation operator$

$f \quad$ Elevation of the surface above the $x$ axis

$f_{\mathrm{f}} \quad$ See Equation $(25)$

$f_{\mathrm{r}} \quad$ See the first paragraph of section 11

$h \quad$ Satellite altitude above the $x$ axis

$k \quad$ Spatial radian frequency

$K$ See Equation $(5)$

$K_{\mathrm{F}}$ See Equation 10

$N$ Number of echoes summed by the altimeter

$p \quad$ Echo power

$\tilde{p} \quad$ See Equation 16

$q \quad$ Transmitted power envelope

$q_{\mathrm{f}} \quad$ Filtered solution to Equation (2)

$t$ Time

$\Delta_{t} \quad$ Temporal sampling interval

$\Delta_{x} \quad$ Spatial sampling interval

$\theta \quad$ Angle subtended at the altimeter by illuminated area

$\sigma \quad$ See sentence following Equation (11)

$\varphi \quad$ See paragraph preceding Equation (18)

$\phi \quad$ See paragraph succeeding Equation (18)

$\gamma \quad$ Radius of area illuminated by the altimeter

$\eta \quad$ See Equation (19)

$\mu \quad$ Signal to noise ratio

$\omega$ Temporal radian frequency

\section{INTRODUGTION}

The measurement of the elevation of ice sheets by satellite altimetry has exploited a theoretical understanding (Brown, 1977) and experimental design (McGoohan, 1975) dating from the 1970s to determine the shape of the sea surface. Ice-sheet elevations, however, have scales of fluctuation absent from the sea surface. In consequence, the present methods of deduction of point elevations from altimeter echoes Brenner and others, 1983; Martin and other, 1983) introduce errors that depend in a complicated fashion on the shape of the surface and the instrument parameters, and which have never been described in any general way. It was observed a long time ago by Laughton (1983) that these limitations might be overcome by changing the way the elevation is deduced from the echoes. In a recent paper (Wingham, 1995; hereafter referred to as (I)), I showed that it was possible with a change in the method used to reduce the echoes to determine the spatial average of the elevation of a region of an ice sheet from satellite altimetry. In this paper, I turn to a consideration of the elevation itself.

The question this paper is concerned with is: what limits the spatial resolution of ice-sheet surface elevations that may be derived from satellite altimeter observations? In common with many geophysical problems, one may suppose this is generally dependent on the spatial and temporal sampling and on the magnitude of random fluctuations. In the treatment here, a two-dimensional, regularly sampled version of the problem is studied theoretically. By restricting the investigation to this case, analytic approximations are possible that allow a simple discussion of the role of sampling and echo fluctuations in limiting the resolution. Some numerical calculations also permit differing surface geometries to be investigated and some idea can be formed of the likely limits of current practical altimeter systems.

The paper introduces in section 3 an approximate expression for the echo that allows a linear equation between the surface elevation and the echo to be 
established. The character of the solution to this equation is discussed in section 4, where it is illustrated that, in the continuous case, the solution is limited by random fluctuations. In consequence, in section 5, a filtered solution is discussed that one anticipates will reduce the effect of fluctuations at the expense of resolution. The way in which sampling affects the resolution is introduced in section 6 . In section 7, some simple expressions are given for the variation of the filtered solution in the presence of echo fluctuations and the balance between sampling-limited and fluctuationlimited resolution is described. Some numerical examples are given in section 8 and it is illustrated that with practical systems the resolution is sampling-limited at around $500 \mathrm{~m}$. This is a considerable improvement on the situation hitherto. In section 9 the inability of the simple treatment given here to account properly for the accuracy of the reconstructed elevations is discussed. Further limitations and extensions of both the model and reconstruction method are described in section 10 . A brief discussion of how our method compares with previous methods and how best these methods may be tested with measurements is given in section 11. Finally, in section 12, I draw some conclusions.

In this paper, detailed derivation of many results is not given. This is partly for the sake of brevity but also because these derivations follow standard routes in the theory of signals and use only standard integrals. Good texts on background signal theory can be found in Proakis and Manolakis (1988) and Papoulis (1965), and a good book of integrals is Gradshteyn and Rhzhik (1980).

\section{A MODEL FOR THE ECHO FROM A ONE-DIMENSIONAL SURFACE}

The general arrangement is shown in Figure 1. A list of symbols is given in section 1. An altimeter lies in the plane $y=0$ at a height $h$ above the $x$ axis. It transmits a pulse $q(t)$ that travels out towards the surface at velocity $c$. The pulse is spatially modulated by the antenna in such a way that the incident power crossing the $x$ axis at the point $x=s$ when the altimeter is at the point $x=v$ varies as $\exp \left[-(v-s)^{2} / \gamma^{2}\right]$. The parameter $\gamma$ determines the spatial extent of the region illuminated by the altimeter, which subtends at the altimeter an angle of order

$$
\theta=\frac{\gamma}{h}
$$

The locus of points at which the power is a maximum as the echo travels outwards is termed the boresight of the antenna. With this particular description, the boresight is normal to the $x$ axis. The intersection of the boresight with the $x$ axis is termed the nadir point. The surface is assumed to vary only with $x$ and the function $f(x)$ is termed the surface elevation. Power echoed from the surface is returned to the altimeter, forming an echo $p$. We assume the surface is homogeneous in its scattering behaviour and that, within the range of angles illuminated by the altimeter, the scattering coefficient is constant. We ignore, for simplicity, energy scattered

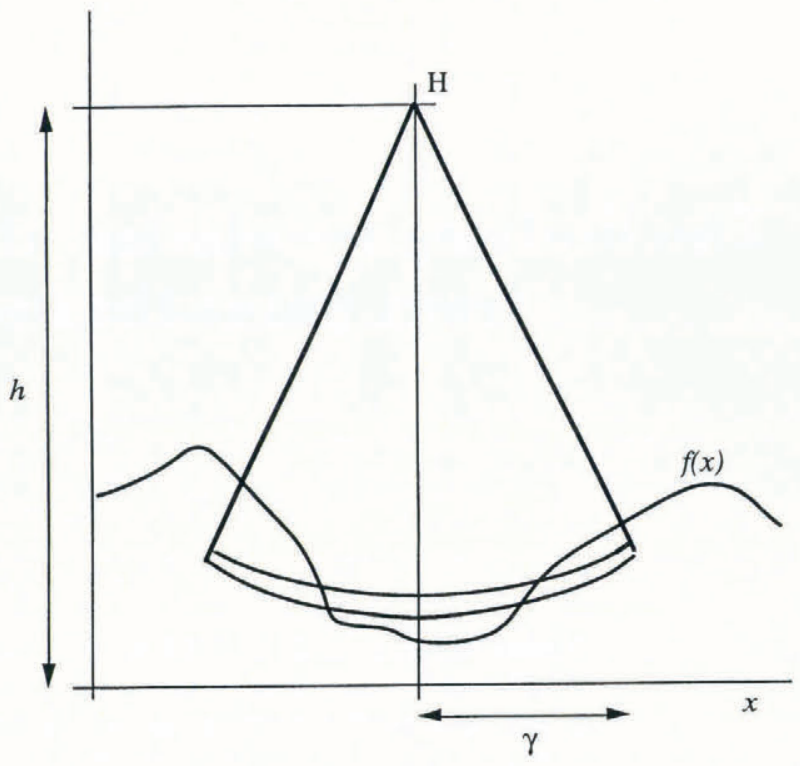

Fig. 1. The geometry of a pulse-limited altimeter measurement over a topographic surface. The altimeter at a point $\mathrm{H}$ and altitude $h$ transmits a pulse that radiates as a thin shell modulated by a beam function that has a radius $\gamma$ on the xaxis. As the shell interacts with the surface, echoes are scattered and some of the energy returns to the satellite. The thickness of the shell is the range resolution of the altimeter. The thickness is small compared with the ranges of the surface illuminated by the beam and, as a result, the echo duration is usually considerably longer than the range resolution.

from beneath the surface although this extension is touched on later.

In (I), I have discussed in considerable detail the application of the Fresnel and related approximations to a general expression for $p$ in the three-dimensional case. Applying these same arguments to the simpler case considered here, one may obtain the non-dimensionalized expression

$$
\begin{aligned}
p(x, t)-\mu^{-1}= & \frac{1}{\pi \sqrt{c h}} \int_{-\infty}^{t} \mathrm{~d} \tau \int_{-\infty}^{\infty} \mathrm{d} s \exp \left[-\frac{c h}{\gamma^{2}}\right] \\
& \cdot\left(t-\tau-\frac{(x-s)^{2}}{c h}\right)^{-\frac{1}{2}} q\left(\tau+\frac{2 f(s)}{c}\right)
\end{aligned}
$$

for the echo $p$ at an altimeter location $x$ and time $t$. In Equation (2), the time origin is taken to be the instant an echo reaches the altimeter from the nadir point. For cases of practical interest, Equation (2) is good to $\mathrm{O}\left(10^{-2}\right)$ in the integrand.

The parameter $\mu$ in Equation (2) is termed the signalto-noise ratio (SNR). The normalization of the echo in Equation (2) is arranged to give it a maximum value of unity when $f=0$ (see Equation (22)), and therefore the terminology SNR is consistent with that of altimeter engineers. Because of this normalization, the backscattering coefficient does not appear explicitly in Equation (2). The parameter $\mu$ accounts only for additive noise and not for the echo fluctuations (termed speckle) which are dealt with in section 7. 


\section{THE SOLUTION OF EQUATION (2); ITS CHARACTER AND IMPLICATIONS}

The elevation $f$ enters non-linearly in Equation (2). However, the elevation may be determined in a linear fashion in the following way. The pulse function $q$ may be defined in such a fashion that

$$
\int_{-\infty}^{\infty} \mathrm{d} t q(t)=1 \text { and } \int_{-\infty}^{\infty} \mathrm{d} t t q(t)=0 .
$$

The lefthand relation of Equation (3) is required for the echo to be of order unity. With Equation (3), one has

$$
f(x)=-\frac{c}{2} \int_{-\infty}^{\infty} \mathrm{d} t t q\left(\tau+\frac{2 f(x)}{c}\right) .
$$

Equation (2) is a linear integral equation for the function $q(\tau+2 f / c)$. If this equation can be solved, it can be solved by a linear operation on the known function $p-\mu^{-1}$. With the function determined this way, the surface elevation may be found using Equation (4).

However, Equation (2) is an integral equation of the first kind. In consequence, the problem is ill-posed; it is not sufficient, for example, for $p-\mu^{-1}$ to be squareintegrable for a solution to Equation (2) to exist. This remains true when one changes the problem in some way, such as the extensions we discuss later; by, for example, extending the analysis to three dimensions, particularizing it to discrete observations of the echo, or restricting the range of time integration in Equation (2). In short, it is fundamental to the problem. This ill-posed nature of the problem has the immediate consequence that, in any practical situation, the error in the solution for $f$ is determined by fluctuations in the observations.

The ill-posed nature of the particular statement of the problem considered here is exposed by developing a formal solution to Equation (2). The kernel of Equation (2) is temporally and spatially shift-invariant and a formal solution of Equation (2) may be developed very simply using Fourier methods. Proceeding this way, one has for the Fourier transform of the kernel in Equation (2)

$$
\begin{aligned}
\frac{1}{\pi \sqrt{c h}} \int_{0}^{\infty} \mathrm{d} t \int_{-\infty}^{\infty} \mathrm{d} x \mathrm{e}^{i \omega t-i k \cdot x} \frac{\exp \left[\frac{-c h}{\gamma^{2}} t\right]}{\left(t-\frac{x^{2}}{c h}\right)^{\frac{1}{2}}} \\
=\frac{c^{2} h^{2} \pi^{2} \gamma^{2}}{\left(c h+i \omega \gamma^{2}\right)} \exp \left[-\frac{c h \gamma^{2} k^{2}}{\left(c h+i \omega \gamma^{2}\right)}\right] .
\end{aligned}
$$

Much can be learned concerning the problem from the character of the function on the righthand side (RHS) of Equation (5), which we now denote by $K$. One has

$$
\begin{array}{rlrl}
K(\omega, k) / K(0,0) & \sim \exp \left[-(\gamma k)^{2}\right] & \omega & \ll \frac{c h}{\gamma^{2}} \\
K(\omega, 0) / K(0,0) & \sim \frac{1}{i \omega} \quad \omega \gg \frac{c h}{\gamma^{2}} \\
K(\omega, k) / K(\omega, 0) & \sim \exp \left[-\left(\frac{c h k}{\omega \gamma}\right)^{2}\left(1+i \frac{\omega}{c h}\right)\right] \\
\omega & \gg \frac{c h}{\gamma^{2}} .
\end{array}
$$

For small $\omega$, one sees from Equation (6) that $K$ is independent of $\omega$ and is simply the Fourier transform of the illumination function. For large $\omega$, one sees from Equation (7) that $K$ decreases as $\omega^{-1}$. From Equation (8), for fixed $\omega$, one sees that $K$ is a Gaussian function modulated with a quadratic phase. The width of this Gaussian function depends on the angle $\theta$ in Equation (1). If one regards an exponential and any product containing one as negligible if the real part of its argument is less than 4 , then an error of $\mathrm{O}\left(10^{-2}\right)$ is introduced which is consistent with the approximation of Equation (2). With this approximation, $K$ may be regarded as negligible outside the region defined by

$$
\frac{c}{2}\left|\frac{k}{\omega}\right|<\theta .
$$

$K$ is restricted to a limited region of the $\{\omega, k\}$ plane lying between the lines given by Equation (9). Energy in the function $q(t+2 f(x) / c)$ that transforms to regions outside this line will be strongly attenuated in the echo field $p$. If one excludes this energy from a solution, one may suppose the error due to fluctuations will be smaller; however, the solution for $q(t+2 f(x) / c)$ will now contain an error as a result of the exclusion, i.e. the solution will approximate a filtered version of $q(t+2 f(x) / c)$, which we shall denote $q_{\mathrm{f}}$.

It is worth pointing out that the limiting of the function $K$ has a simple physical explanation. A plane wave, travelling with velocity $c$, whose wave front makes an angle $\theta$ with the $x$ axis maps to a point in the $\{\omega, k\}$ plane lying on one of the two lines given by Equation (9). The limiting of $K$ arises from the limited angular illumination of the altimeter; one supposes, then, that the (albeit non-linear) effect on the echoes of variations of elevation making angles larger than $\theta$ with the $x$ axis will be strongly attenuated. We shall illustrate later that this is indeed the case.

\section{A FILTERED SOLUTION FOR $q(t+2 f(x) / c)$}

The problem is therefore one of filter design. Were one to treat it as a problem of optimal design, then it is a complicated one, partly because Equation (2) is nonlinear in the unknown, and partly because a principal fluctuation contaminating altimeter echoes is non-linear in the known, as we discuss later. However, my purpose here is to explore the problem, and I wish to keep the treatment simple and analytic as far as possible. Therefore, I shall introduce a known filter which serves at least for the purpose of illustration. More general approaches will be discussed later.

Rather than operate on $p-\mu^{-1}$ with the inverse of $K$, I shall use instead a filter $K_{\mathrm{F}}$ defined to satisfy

$$
K_{\mathrm{F}}(\omega, k) K(\omega, k)=\exp \left[-\frac{\omega^{2}}{\sigma^{2}}-\frac{2 c^{2} h^{2} \gamma^{2} k^{2}}{\left(c^{2} h^{2}+\omega^{2} \gamma^{4}\right)}\right] .
$$

$K_{\mathrm{F}}$ differs from the inverse $K^{-1}$ by the function on the RHS of Equation (9). This function is the filter that, acting on $q(t+2 f(x) / c)$ results in the function $q_{\mathrm{f}}$. With our approximation concerning exponentials, it makes 
negligible the spectrum of $q_{\mathrm{f}}$ in those regions of the $\{\omega, k\}$ plane lying outside the region defined by Equation (9) and

$$
|\omega|<2 \sigma \text {. }
$$

The parameter $\sigma$ is a free parameter that determines the temporal band width of $q_{\mathrm{f}}$. Its value we shall discuss in the next section.

In broad terms, it is apparent from our discussion of the behaviour of $K$ that $K_{\mathrm{F}}$ attenuates energy of the spectrum of $q(t+2 f(x) / c)$ that is poorly represented in the echoes.

\section{THE CONSEQUENGES OF SAMPLING}

We now consider the consequences on the reconstruction of regularly sampling the echo in time and space. An advantage of restricting the treatment to two dimensions is that this may be a reasonable approximation to the practical situation. Sampling has two consequences: it introduces the possibility of non-uniqueness into the reconstruction and it determines the importance of echo fluctuations on the calculation of $q_{\mathrm{f}}$. In this section, we deal with the first of these; in the next section we deal with the fluctuations.

As is well known from sampling theory, a function may be reconstructed exactly from its regularly sampled form, provided it satisfies Shannon's sampling theorem. In the present case, one may state this as follows: if the echo is sampled with temporal and spatial sampling intervals $\Delta_{t}$ and $\Delta_{x}$ respectively, it may be determined uniquely provided its spectrum falls entirely in the box centred on the origin in the $\{\omega, k\}$ plane defined by the equations

$$
\omega= \pm \frac{\pi}{\Delta_{t}}
$$

and

$$
k= \pm \frac{\pi}{\Delta_{x}} .
$$

For most practical purposes, the temporal behaviour of the function $q$ can be regarded as satisfying this theorem. Since Equation (2) describes a temporally shift-invariant operation on $q$, this is true of the echo too.

The echo spectrum is the product of the spectrum of the function $q(t+2 f(x) / c)$ with the function $K$, and therefore the echo spectrum is also limited by the behaviour of $K$, in addition to that of $q$. We have determined that $K$ is limited to the region falling between the lines described in inequality (9). One may therefore also assume that the spectrum of the echo is negligible outside the region defined by Equation (12) and

$$
\frac{c}{2}\left|\frac{k \Delta_{t}}{\pi}\right|<\theta .
$$

Thus, were one to choose the spatial sampling interval to satisfy $\Delta_{x}<c \Delta_{t} /(2 \theta)$, the echo may be regarded as satisfying Shannon's theorem in both dimensions.

Practical altimeter systems satisfy this requirement. In addition, however, an altimeter sums each group of $N$ consecutive spatial samples and desamples the result. (In fact, the altimeter summation introduces time shift between each spatial sample in the sum. We assume here the effects of these shifts are negligible.) The desampled spatial sampling interval $N \Delta_{x}$ is typically larger than $c \Delta_{t} /(2 \theta)$ and the situation arises that is illustrated in Figure 2. Generally, it will not be possible to determine the echo spectrum uniquely. However, as Figure 2 illustrates, there remains in this case a region of the $\{\omega, k\}$ plane defined by inequalities $(9)$ and

$$
|\omega|<\frac{c \pi}{2 N \Delta_{x} \theta}
$$

in which the echo spectrum may be uniquely determined. This region is shown hatched in Figure 2. A numerical example may help to illustrate this. For the parameters given in section 7, taken as describing the ERS-1 altimeter in its ice mode of operation, the parameter $c \Delta_{t} /(2 \theta) \sim 100$, while $\Delta_{x}$ equals 5 , and one could take Shannon's criteria as satisfied. On the others hand, $N \Delta_{x}$ equals 250 and, in general, the desampled echo field will be aliased.

This behaviour may be described using the results of sampling theory. One has that the function

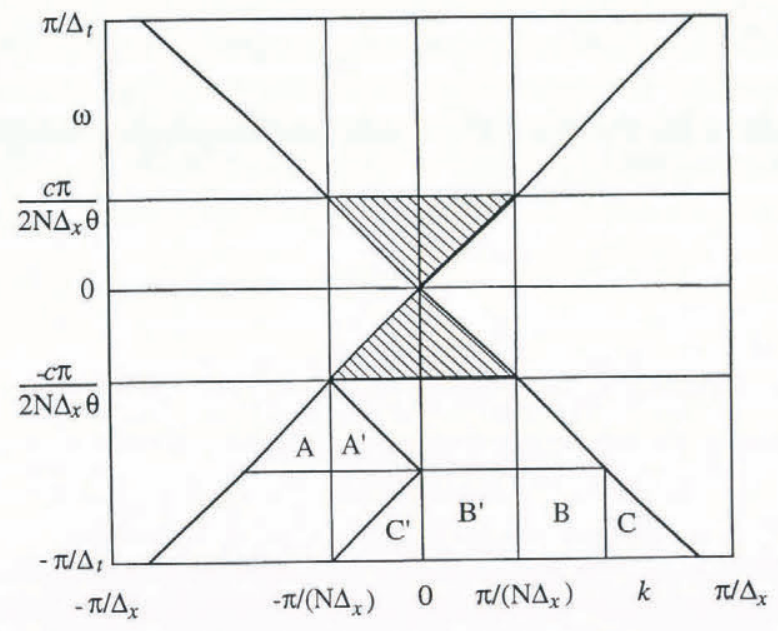

Fig. 2. The effect of sampling on the spectrum of the altimeter echo. In practice, the echo spectrum may be regarded as negligible outside of the box in the $\{\omega, k\}$ plane bounded by the Nyquist frequencies in time and space. This is because the spectrum is negligible outside the region described by inequality (9) and is limited by the transmitted pulse to lie within the temporal Nyquist frequency $\pi / \Delta_{t}$. The spatial desampling of the altimeter echo will lead generally to aliasing about the spatial frequency $\left(\pi / N \Delta_{x}\right)$. For example, the energy in the triangular region $\mathrm{A}$ will be mapped to energy in the triangle $\mathrm{A}^{\prime}$, reclangle $\mathrm{B}$ to $\mathrm{B}^{\prime}$ and triangle $\mathrm{C}$ to $\mathrm{C}^{\prime}$ and so on. However, a limited region of the plane, shown hatched in the figure, remains unaffected by aliasing. If the echo spectrum is limiled to this region, with a filter that attenuates temporal frequencies outside the region $|\omega| \leq c \pi /\left(2 N \Delta_{x} \theta\right)$, Shannon's resampling theorem may be applied to the filtered spectrum. Note that the figure is not to scale. 


$$
\begin{aligned}
\tilde{p}(t, x)= & \Delta_{x} \Delta_{t} \sum_{n=-\infty}^{\infty} \frac{\sin \left(\pi\left(x-n \Delta_{x}\right) / \Delta_{x}\right)}{\pi\left(x-n \Delta_{x}\right)} \\
& \cdot \sum_{m=-\infty}^{\infty} p\left(m \Delta_{t}, n \Delta_{x}\right) \frac{\sin \left(\pi\left(x-m \Delta_{t}\right) / \Delta_{t}\right)}{\pi\left(x-m \Delta_{t}\right)}
\end{aligned}
$$

has a spectrum negligibly different to that of $p$ in the region centred on the origin and bounded by inequalities (9) and (15).

We have noted that the spectrum of $q_{\mathrm{f}}$ is limited by the parameter $\sigma$ to the region described by inequalities $(9)$ and (11). If one chooses the parameter $\sigma$ to satisfy

$$
\sigma \leq \frac{c \pi}{4 N \Delta_{x} \theta}
$$

then on substituting Equation (17) into Equation (11) and comparing the result with Equation (15), one sees that the spectrum of $q_{\mathrm{f}}$ is limited to the same region bounded by inequalities (9) and (15) on which the spectra of $p$ and $\tilde{p}$ may be regarded equal. It then follows that the result of filtering $\tilde{p}$ with $K_{\mathrm{F}}$ is negligibly different from that of filtering $p$ with $K_{\mathrm{F}}$. With the proviso of Equation (17), then, one may replace $p$ with $\tilde{p}$ in determining the filtered solution $q_{\mathrm{f}}$.

One does not have to acknowledge explicitly the desampling in determining $\tilde{p}$ via Equation (16). This is because, with the restriction of Equation (17), $K_{\mathrm{F}}$ spatially filters the echoes. In doing so, it accounts (with minor differences from the practical situation) for the summation of the echoes and makes the spectrum of $q_{\mathrm{f}}$ negligible outside the region $|k| \leq \pi /\left(N \Delta_{x}\right)$. With the latter accomplished, it is then theoretically immaterial whether one uses $p\left(t, \Delta_{x}\right)$ or $p\left(t, N \Delta_{x}\right)$ to reconstruct $\tilde{p}$. We use $p\left(t, \Delta_{x}\right)$ because it is then unnecessary in the next section to treat the fluctuation reduction resulting from the summation of the echoes separately from the reduction resulting from filtering the echoes with $K_{\mathrm{F}}$.

\section{THE CONSEQUENCES OF NOISE}

An altimeter echo is a random fluctuation. The expression given in Equation (2) is the expectation of this fluctuation. The interpolated function $\tilde{p}$ is also the expectation of a process $\varphi$ derived from replacing the mean echo $p$ with the random fluctuation in Equation (16); that is

$$
E\{\varphi\}=\tilde{p}
$$

If one determines a function $\phi$ by operating on $\varphi-\mu^{-1}$ with the filter $K_{\mathrm{f}}$, it too will fluctuate randomly. Since $K_{\mathrm{f}}$ is linear, one has from the result of the previous section that the expectation of $\phi$ is the filtered solution $q_{\mathrm{f}}$. The magnitude of the fluctuations of $\phi$ can be characterized by the number $\eta$ defined by

$$
\eta^{2}=\frac{q_{\mathrm{f}}^{2}}{E\left\{(\phi-E\{\phi\})^{2}\right\}}
$$

A small value of $\eta$ tells one that the operation of $K_{\mathrm{f}}$ on $\varphi-\mu^{-1}$ will teach us little concerning $q_{f}$; it will be submerged in fluctuations.

To determine the number $\eta$, one needs the correlation function of the sampled echo. In practice, the sampling intervals of an altimeter are chosen by design (Walsh, 1982) to make

$$
\begin{aligned}
& E\left\{\varphi\left(x_{i}, t_{j}\right) \varphi\left(x_{n}, t_{m}\right)\right\} \\
& \quad=\left(\tilde{p}\left(x_{i}, t_{j}\right)\right)\left(\tilde{p}\left(x_{n}, t_{m}\right)\right)\left(1+\delta_{i n} \delta_{j m}\right)
\end{aligned}
$$

a good approximation to the correlation function when $\varphi-\mu^{-1}$ is exponentially distributed, and the altimeter echoes from ice sheets have statistics that are closely exponential. The difficulty of determing $\eta$ or any other measure of the fluctuations of $\phi$ ) is exposed by Equation (20): the fluctuations of $\phi$, which depend on the correlation function in Equation (20), are non-linearly dependent on $p$ (since, from Equation (16), $\tilde{p}=p$ at the sample instants), which is, in turn, non-linearly dependent on the elevation $f$. This makes a general theoretical exploration of the behaviour of $\eta$ difficult.

The only way of making some analytic progress whilst retaining a rough idea of the behaviour of the fluctuations is to replace $\tilde{p}$ in Equation (20) with a constant. Since $p$ is arranged by definition to be of order unity, we set this constant equal to $1+\mu^{-1}$. With this replacement, the correlation function in Equation (20) is stationary and one has from well-known results in sampling and random variable theory that

$$
\begin{gathered}
E\left\{(\phi-E\{\phi\})^{2}\right\}=\frac{\Delta_{t} \Delta_{x}\left(1-\mu^{-1}\right)^{2}}{4 \pi^{2}} \int_{-\pi / \Delta_{t}}^{\pi / \Delta_{t}} \mathrm{~d} \omega \\
\cdot \int_{-\pi / \Delta_{x}}^{\pi / \Delta_{x}} \mathrm{~d} k K_{\mathrm{F}}(\omega, k) \bar{K}_{\mathrm{F}}(\omega, k) \\
\sim \frac{\Delta_{t} \Delta_{x}\left(1-\mu^{-1}\right)^{2}}{4 \pi^{2}} \int_{-\infty}^{\infty} \mathrm{d} \omega \int_{-\infty}^{\infty} \mathrm{d} k K_{\mathrm{F}}(\omega, k) \bar{K}_{\mathrm{F}}(\omega, k) \\
\sim \frac{\sqrt{\pi} \Delta_{t} \Delta_{x} \sigma^{4}\left(1-\mu^{-1}\right)^{2} \gamma}{4 \sqrt{2} \pi^{6}(c h)^{5}} \quad \frac{c h}{\sigma \gamma^{2}} \ll 1
\end{gathered}
$$

where the first approximation follows because we have assumed that $K_{\mathrm{F}}$ is negligible outside the limits of the first integral on the RHS, and the second uses the definition Equation $(10)$ of $K_{\mathrm{F}}$ and ignores terms in the stated small parameter. This is a good approximation for practical systems.

To give an expression for $\eta$ one needs to determine the filtered solution $q_{\mathrm{f}}$. This again is difficult to do in any general way because the elevation enters Equation (2) non-linearly. Since we have approximated roughly the denominator on the RHS of Equation (19), we will deal with the numerator in the same spirit and consider the value of $\eta$ when the elevation $f$ is identically zero. How one may understand the result we then obtain for $\eta$ in a more general context will be discussed by example later. In this simple case, Equations (2) and (16) simplify to 


$$
\begin{gathered}
\tilde{p}(x, t) \equiv p(t)=\mu^{-1}+\int_{-\infty}^{t} \mathrm{~d} \tau \exp \left[-\frac{c h(t-\tau)}{\gamma^{2}}\right] q(\tau) \\
f \equiv 0 \\
\sim \mu^{-1}+\left\{\begin{array}{cc}
0 & t<0 \\
\exp \left[-\frac{c h t}{\gamma^{2}}\right] & t \geq 0 .
\end{array}\right.
\end{gathered}
$$

The approximation of the second line assumes the duration of pulse function $q$ short in comparison with that of the echo. This is characteristic by definition of pulse-limited altimetry. With Equation (22) and the definition Equation $(10)$ of $K_{\mathrm{F}}$, one obtains

$$
q_{\mathrm{f}}(t)=\frac{\sigma}{2\left(2 \pi^{2} c h \gamma^{2}\right)^{2}} \exp \left[-\frac{t^{2} \sigma^{2}}{4}\right] .
$$

When $f \equiv 0$, the surface is plane and coincides with the $x$ axis. Equation (23) describes a function independent of $x$, symmetric about the $x$ axis. Its maximum coincides with $t=0$. Substituting from Equations (21) and (23) into Equation (19) then yields

$$
\eta^{2}=\frac{\pi c h}{8 \sqrt{2 \pi} \Delta_{t} \Delta_{x}\left(1-\mu^{-1}\right)^{2} \sigma^{2} \gamma} \quad t=0
$$

If Equation (17) is satisfied with equality, $\sigma$ may be eliminated from Equation (24), and the result given in terms of altimeter-operating parameters alone.

Equation (24) is subject to an important proviso, other than the limitations of the assumptions leading to its derivation. In practice, spatial and temporal sampling intervals are chosen by design to be the smallest possible that allow the approximation Equation (20) of the correlation function. One is not free to make $\Delta_{t}$ and $\Delta_{x}$ as small as one needs to make $\eta$ as large as one wishes, which is the implication of Equation (24). In fact, for a given altimeter design and orbit altitude, the only free parameter available to increase the value of $\eta$ is $\sigma$.

Making the parameter $\sigma$ smaller, however, does not only change $\eta$. As we noted in section $6, \sigma$ also determines the spatial band width. Generally speaking, the spatial resolution is limited to the reciprocal of this band width. If Equation (17) is satisfied with equality, so that $\sigma$ takes its maximum value, the reciprocal spatial band width is $2 N \Delta_{x}$. If $\sigma$ is made smaller, to reduce the effect of echo fluctuations on $q_{\mathrm{f}}$, then one may anticipate the spatial resolution will increase in proportion.

It is the character of the continuous problem that the resolution is fundamentally limited by the echo fluctuations. When the echo field is sampled, this view needs to be modified, because the spatial-sampling interval also limits the resolution. In the practical situation, one or other of these processes may dominate. If Equation (17) can be satisfied with a usefully large value of $\eta$, one may regard the spatial resolution as sampling-limited. On the other hand, if one needs a smaller value of $\sigma$ to obtain an adequate value of $\eta$, one may regard the spatial resolution as fluctuation-limited.

\section{NUMERICAL RESULTS}

The discussion of section 7 gives some insight into the effect of echo fluctuations on the reconstruction. However, the problem dealt with there is trivial to the extent that were it the case that $f \equiv 0$ there would be no cause to improve the spatial resolution. This is the case in practical altimetry of the ocean surface and some limited areas of ice sheets. When topography is present, one needs to have a more general idea of the effect of sampling and fluctuations, and in this section we look at some numerical examples.

Satellite altimeters orbit the Earth at altitudes $h$ of around $1000 \mathrm{~km}$. They illuminate an area with a radius $\gamma$ of $10 \mathrm{~km}$ or so. The temporal sampling interval $\Delta_{t}$ of the radar may vary but we will take $10 \mathrm{~ns}$. The spatial sampling interval $\Delta_{x}$ varies but $5 \mathrm{~m}$ is typical. It has become customary to have designs with $N$ equal to 50 . This selection of parameters may be taken as describing, for example, the ERS-1 altimeter in its ice mode of operation. In the following examples, we use these numerical parameters.

Figure 3 shows the reconstruction $q_{\mathrm{f}}$ of a square wave surface. To obtain this figure and the next, Equation (2) was used to calculate the echo, Equation (10) to define $K_{\mathrm{F}}$, Equation (16) to determine $\tilde{p}$ and Equation (17) was satisfied with equality. The surface has a wavelength of $10 \mathrm{~km}$ and an amplitude of $20 \mathrm{~m}$. In this figure and succeeding ones, the time coordinate has been converted to an equivalent elevation. The overlap between the upper and lower surfaces occurs over a kilometre or so. The spatial resolution $2 N \Delta_{x}$ is $500 \mathrm{~m}$ for this example. For this illustration, the reciprocal spatial band width underestimates somewhat the horizontal resolution. This is not surprising since, as Figure 2 illustrates, most of the energy in the spectrum of $q_{\mathrm{f}}$ is limited to a spectral band width rather less than $\pi / N \Delta_{x}$.

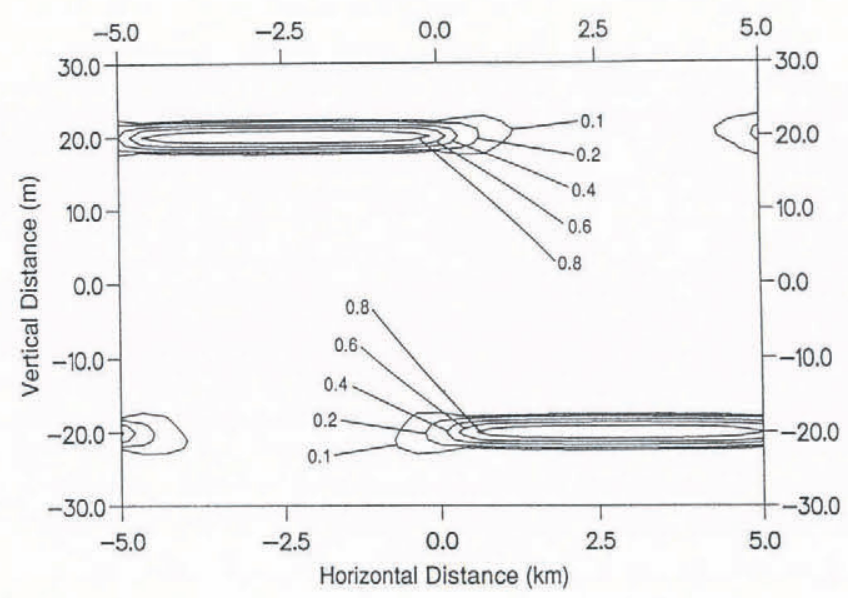

Fig. 3. The function $q_{\mathrm{f}}$ reconstructed from a square-wave surface with an amplitude of $20 \mathrm{~m}$ and a wavelength of $10 \mathrm{~km}$. The instrument parameters used for this illustration are given in the text. The amplitude of $q_{\mathrm{f}}$ is normalized to a maximum value of unity and the scale is linear. The figure illustrates that the edges of the reconstructed square wave are more gradual than the $500 \mathrm{~m}$ spatial band width suggests. 
With the values we have assumed, the range of illumination angles is $0.57^{\circ}$. As we remarked at the end of section 4, one may anticipate that surfaces with gradients larger than this angle will be poorly imaged. In Figure 4 we show the result of migrating a sinusoidal surface with an amplitude of $20 \mathrm{~m}$ and a wavelength of $10 \mathrm{~km}$. The maximum gradient of the surface is $0.72^{\circ}$. The parts of the surface with highest gradients are not imaged in Figure 4. The contours in the region of higher gradients break up due to interference from the reconstruction of the lower-gradient regions.

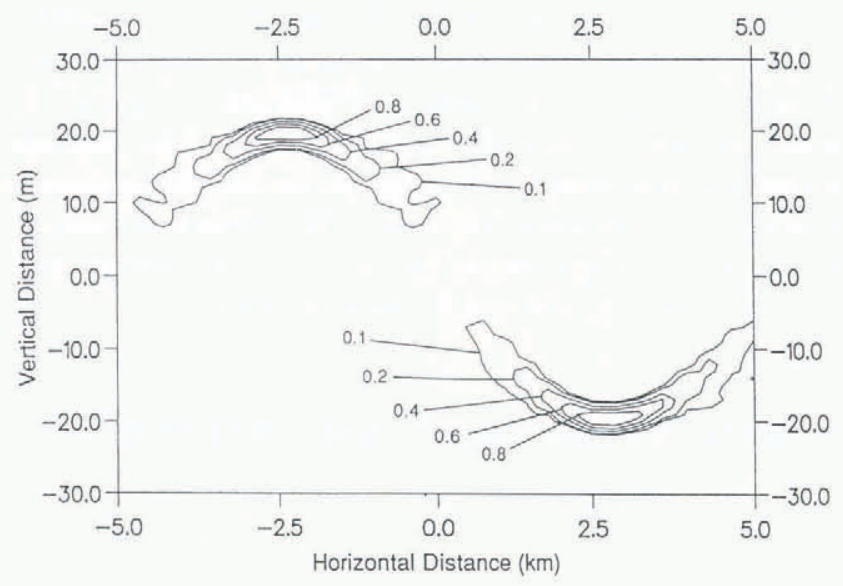

Fig. 4. The function $q_{\mathrm{f}}$ reconstructed from a sine-wave surface with an amplitude of $20 \mathrm{~m}$ and a wavelength of $10 \mathrm{~km}$. The instrument parameters used for this illustration are given in the text. The amplitude of $q_{\mathrm{f}}$ is normalized to a maximum value of unity and the scale is linear. The figure illustrates how the elements of the surface with gradients larger than half the range of illuminated angles are poorly imaged by the altimeter and are lost in the effects from the parts of the surface with lower gradients.

The failure to image higher gradients is not a result of the procedure. Pulse-limited altimeters are fundamentally insensitive to slopes outside their range of illumination angles. If any method is used to generate continuous surfaces from altimeter echoes, the location of the surface when its gradient is larger than the range of illumination angles is an assumption of the method.

Figures 3 and 4 were computed by taking the spatial resolution to be sampling-limited. Is this in fact the case for these examples? Taking $c$ as $3 \times 10^{8} \mathrm{~ms}^{-1}$ and the $\mathrm{SNR}$ as $\infty$ (that is, assuming the speckle fluctuations are the dominant source of randomness), the simple calculations of section 7 give a value of $\eta$ of 33 from Equations (24) and (17). Figure 5 shows the result of an exact calculation of the function $\eta$ for the square-wave surface whose reconstruction is shown in Figure 3. The peak value of $\eta$ in Figure 5 is 42 whereas our approximation is 33. The contours of $\eta$ are similar to the contours of the signal in Figure 3, except that in Figure 3 the negative cycles of the signal are hidden. The noise is approximately stationary but in detail one notices that the higher parts of the surface have higher values of $\eta$. The signal term in Figure 3 does not show this dependence and so it is associated with the fluctuations. It is not hard to explain.

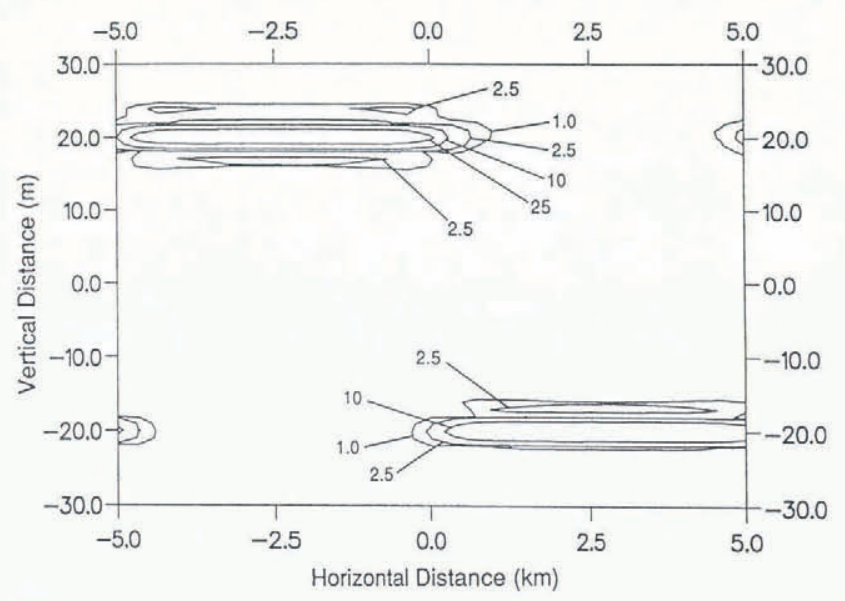

Fig. 5. The function $\eta$ for the reconstruction of the square wave shown in Figure 3. The paramelers used for this illustration are the same as in Figure 3. The negative cycles of the migration are suppressed in Figure 3 but appear positive in the function $\eta$. With this difference, comparison of this figure with Figure 3 illustrates that the noise is approximately stationary, although a small dependence on vertical elevation is apparent.

The echoes from lower elements of the surface have higher levels of fluctuation than our simple estimate assumes, because they have additional contributions from echoes from higher elements of the surface. The converse is true for the echoes from the higher elements of the surface. Thus, we may anticipate that higher elements of the surface have rather higher values of $\eta$ than our simple expressions describe and lower parts of the surface rather lower values of $\eta$. This is what we see in Figure 5. In the case of the square-wave surface, the resolution is sampling-limited.

However, the approximations of section 7 describe a situation where the surface has low gradients in regions of greater extent than the horizontal resolution. The function $\eta$ decreases rapidly as the slope increases due to the signal loss that is illustrated in Figure 4 and here the approximations of section 7 are less useful. Figure 6 shows the result of an exact calculation of the function $\eta$ for the reconstruction of the sinusoidal surface. The contours of $\eta$ coincide approximately with the signal contours of Figure 4 and the high-low asymmetry of Figure 5 is also apparent. However, the maximum value of $\eta$ in Figure 6 is around half that of Figure 5. The higher-slope regions of the surface disappear into the noise at slightly smaller slopes than they disappear in Figure 4. For this example, the ability to image slopes is fluctuation-limited. One sees then that the importance of sampling and fluctuations in determining the resolution is not quite straightforward in the practical situation; it will depend on the character of the surface.

\section{A SOLUTION FOR THE HEIGHT; A LIMIT- ATION OF THE PRESENT TREATMENT}

One is principally interested in the elevation $f$. Thus far, we have only considered an approximate solution for the function $q(\tau+2 f / c)$. What is the relationship between 
the elevation $f$ and the approximate solution $f_{\mathrm{f}}$ resulting from the approximation $q_{\mathrm{f}}$ ? Ignoring the effect of fluctuations, this relation can be obtained by replacing $q$ with $q_{\mathrm{f}}$ in Equation (4):

$$
\begin{aligned}
f_{\mathrm{f}}(x)= & -\frac{c}{8 \pi^{2}} \int_{-\infty}^{\infty} \mathrm{d} t t \int_{-\infty}^{\infty} \mathrm{d} \tau \int_{-\infty}^{\infty} \mathrm{d} s \\
& \cdot q\left(t-\tau+\frac{2 f(x-s)}{c}\right) \int_{-\infty}^{\infty} \mathrm{d} \omega \mathrm{e}^{+i \omega \tau} \int_{-\infty}^{\infty} \mathrm{d} k \mathrm{e}^{+i k s} \\
& \cdot K_{\mathrm{F}}(\omega, k) K(\omega, k) \\
= & \frac{1}{2 \sqrt{2 \pi} \gamma} \int_{-\infty}^{\infty} \mathrm{d} s f(x-s) \exp \left[-\left(\frac{s}{2 \sqrt{2} \gamma}\right)^{2}\right]
\end{aligned}
$$

This equation shows that the mean filtered solution $f_{\mathrm{f}}$ is the result of smoothing the elevation $f$ with a filter whose spatial extent is larger by a factor $2 \sqrt{2} \gamma$ than the region illuminated by the altimeter (cf. the description of the antenna modulation in the paragraph preceding Equation (1)). This would mean, for example, that in the case of the illustrations of the previous section, the spatial resolution of the elevation derived from the functions shown in Figures 3 and 4 was $28 \mathrm{~km}$. Yet, Figures 5 and 6 clearly show the function $q_{\mathrm{f}}$ can be resolved to a kilometre or so. Why is the spatial resolution implied by Equation (25) so much larger than that deduced from the spatial band width?

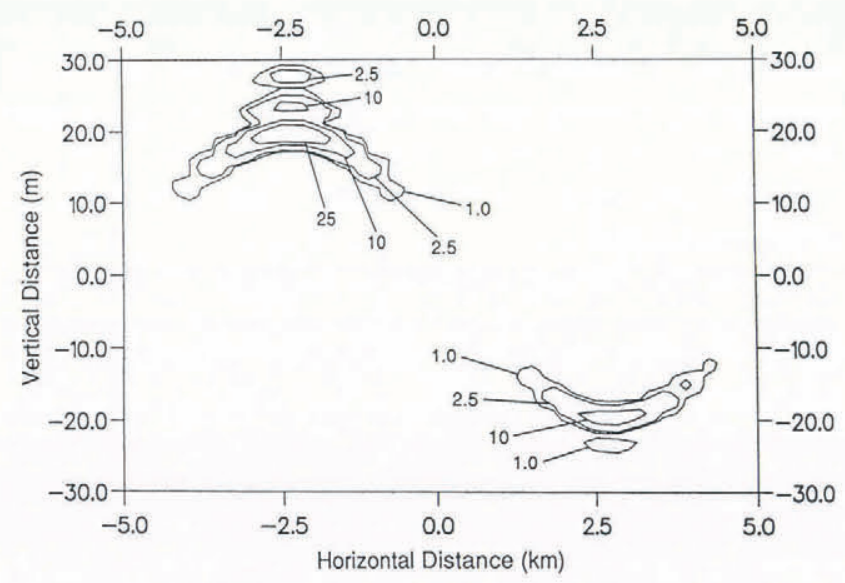

\begin{abstract}
Fig. 6. The function $\eta$ for the reconstruction of the sine wave shown in Figure 4. The parameters used for this illustration are the same as in Figure 4. The negalive cycles of the migration are suppressed in Figure 4 but appear positive in the function $\eta$. Comparison of this figure with Figure 4 shows that the visibility of the poorly imaged gradients of the surface is further restricted by the fluctuations.
\end{abstract}

The answer to this question lies in the range of integration of the $t \mathrm{~d} t$ operator in Equations (4) and (25). We have assumed this infinite and we have done this to allow the use of simple Fourier techniques. Figures 3 and 4 show the function $q_{\mathrm{f}}$ over a finite interval, but in fact it is not zero outside this interval and, when this energy is included into the integral over $t$ in Equation (25), the result is to reduce the resolution. (This argument can be made quantitative by considering the inverse transform of the RHS of Equation (10) but we shall not pursue this here.)

It is perfectly clear that one may determine the surface elevation in Figures 3 and 4 with a resolution better than implied by Equation (25). The reason this is the case is that one is prepared to make an a priori assumption concerning the approximate location of the surface. Mathematically, this assumption can be included by restricting the time interval in Equation (2) on which $q(\tau+2 f / c)$ is defined, that is, restricting the domain of the operator $K$. This done, the problem can no longer be diagonalized using Fourier methods and one needs to revert to a more general linear method. This is the approach taken in Wingham in press; (I) here) for determining the average height. The analytic simplicity and insight afforded by the simple approach taken here is then lost. However, this extension seems to be necessary to determine the accuracy of a reconstruction of $f$ but this will not be pursued here.

\section{OTHER LIMITATIONS AND EXTENSIONS}

We now comment on other limitations and possible extensions of the method. Some of these have been discussed at length in (I) and we simply mention them here. The forward model is subject to a number of assumptions concerning the geometry and scattering behaviour. An extension of $K$ to include simple volumescattering would follow from a straightforward carry over from (I). The theory in (I) does not consider in any detail the relation between the electromagnetic surface and the physical one and this weakness carries over here. This will certainly lead to difficulties in regions of seasonal melting. One could also carry over from (I) the extension to the case where the antenna boresight is not normal to the datum plane. Both these extensions could be dealt with by the Fourier methods used here but one would need, particularly in the case of a non-normal boresight, to reconsider the choice of $K_{\mathrm{F}}$ because $K$ will be significantly different, and one should expect significantly different behaviour of $\eta$.

One may also extend slightly the range of surfaces considered here by introducing a plane datum that includes a finite slope across the satellite track (that is, in the $y$ direction of our coordinate system). In fact, the analysis is very similar to that of a non-normal boresight.

However, one properly needs to deal with the threedimensional reconstruction of two-dimensional surfaces. In three dimensions, however, one has to accept that, in any case of practical interest, the spatial sampling is irregular. While it is at least possible that in some circumstances the method described here may be practically useful, this would not be the case were one to extend the present calculations to a rectangular grid of samples, although this is easily done. The presence of irregular sampling alters the case theoretically and practically. Theoretically, it introduces a uniqueness problem that we have avoided here by restricting the spectrum of the echoes to satisfy Shannon's theorem. It 
also remains possible to retain in a formal way the distinction between sampling-limited and noise-limited resolution, although no longer with Fourier techniques, but it is arguable whether such measures can be practically interpreted simply; at the least, the resolution will become position-dependent. In practice, irregular sampling alters the case considerably and one needs more general linear methods, such as least-squares or minimum-norm methods. These considerably increase the computations needed, because the problems cannot be a priori diagonalized. It remains to find a theoretically acceptable but practically sensible threedimensional implementation. However, I anticipate that the two-dimensional analysis here will greatly aid an understanding of a three-dimensional reconstruction from irregular samples.

We have not considered here the consequences of the truncation of the echoes by the altimeter-recording system. Once one has restricted the domain of $K$ along the lines discussed in section 9 , it is a simple matter to include a restriction of the range of $K$, which has the effect of truncating the echoes. In fact, as showed in (I), this modification is essential for a proper consideration of the uniqueness of any reconstruction, although this is not pursued further here.

\section{RELATIONSHIP TO OTHER METHODS; EXPERIMENTAL VERIFICATION}

Techniques that have been applied previously to altimeter echoes from ice sheets are separable, that is, they proceed by functionals that are respectively independent of $x$ and independent of $t$, termed the "retracker" (Martin and others, 1983) and "slopeinduced error correction" (Brenner and others, 1983), the result $f_{\mathrm{r}}(x)$ of which is supposed to describe the surface $f(x)$. There is not general agreement concerning the detailed implementation of these procedures (see e.g. Remy and others, 1989; Bamber, 1994). However, as was pointed out in some detail in $(\mathbf{I})$, there is no theoretical basis for using a separable procedure and one must therefore generally suppose that the solution $f_{\mathrm{r}}(x)$ generated by such a procedure is not in fact equal to the $f(x)$. This observation is probably of greatest practical importance in determining changes in the surface with repeated observations, since generally the function $f_{\mathrm{r}}(x)$ has dependencies on instrument parameters and attitude. However, these dependencies will also be present in a derived topography.

Remy and others (1989) have considered the interpolation problem, that is, creating a two-dimensional surface from a finite set of erroneous point observations of the function $f_{\mathrm{r}}(x)$. In their treatment, Remy and others did not distinguish between the functions $f_{\mathrm{r}}(x)$ and $f(x)$. Their description of the procedure as an inversion is therefore erroneous. Were continuous observations of the function $f_{\mathrm{r}}(x)$ available, their treatment would leave only an estimation problem to be solved; with the distinction between $f(x)$ and $f_{\mathrm{r}}(x)$ acknowledged, the inversion problem still remains. I would also speculate, on the basis of the results here, that the limiting resolution will not be achieved with a separable procedure - Equation (25), for example, can be taken as describing a separable procedure and we have noted the poor resolution that results. To be clear, however, I do not wish to suggest that the threedimensional interpolation problem is not an essential component of the problem or that the sampling density is not at present a limiting-error source in the use of existing altimeter observations.

Turning to considering the experimental confirmation of our results. It is easy enough to take a section of altimeter echoes and migrate them with the methods described here. Indeed, this has already been reported on (Wingham and others, 1993). However, this exercise must necessarily produce a surface consistent with the echoes and it does not follow that the reconstructed surface coincides with the ice surface. At the kilometrelength scales at which solving the inversion problem may be practically important (i.e. at which $f(x)$ and $f_{\mathrm{r}}(x)$ may differ significantly), the topography of ice sheets is three-dimensional. One generally needs to solve the problem fully to make a comparison with ground observations and these need to be accurate high-density observations in two-dimensions of the surface elevation coincident with the altimeter observations. The equipment to make such geodetically accurate surveys is now becoming available in the United States and Europe with the use of GPS-tied airborne-laser altimeters. The data provided by these instruments should permit an opportunity to verify the results presented here.

\section{GONGLUSIONS}

This paper provides a theoretical description of a method improving the spatial resolution of ice-sheet elevations derived from satellite-altimeter echoes from ice sheets, with a view to determining the fundamental limits on this resolution. It shows that, in two dimensions at least, there is sufficient information content in successive echoes that their spatial resolution may be improved without a catastrophic degradation due to echo fluctuations. The method shows that the spatial resolution may theoretically be limited either by the echo sampling. In present practical circumstances, it is the spatial sampling that limits the resolution, although this conclusion may not carry over to a three-dimensional treatment. The spatial resolutions indicated by the numerical calculations here are of the order of $500 \mathrm{~m}$, which is very considerably smaller than thought possible hitherto. Since much of the local topography of ice sheets is at horizontal scales of 1 $20 \mathrm{~km}$, this is a useful advance. A practical demonstration of the method has been given (Wingham and others, 1993 and this paper justifies the statements made there concerning the method.

Important issues remain to be dealt with. Principal among these are the determination of the accuracy of the retrieved elevations, which in my view requires an investigation of solutions that limit the vertical range of the elevations. A three-dimensional implementation that is practically sensible is also needed and is probably necessary before making useful comparisons of the results with ground observations. 


\section{ACKNOWLEDGEMENT}

This work was in part supported by the U.K. Science and Engineering Research Council, under grant GR/G46646, and by the U.K. Natural Environment Research Council, under grant GR3/8748.

\section{REFERENCES}

Bamber, J. L. 1994. Ice sheet altimeter processing scheme. Int. F. Remole Sensing, $15(4), 925-938$.

Brenner, A. C., R.A. Bindschadler, R. H. Thomas and H.J. Zwally. 1983. Slope-induced errors in radar altimetry over continental ice sheets. F. Geophys. Res., 88 C3), 1617-1623.

Brown, G.S. 1977. The average impulse response of a rough surface and its application. IEEE Trans. Antennas Propag., AP-25, 67-74.

Gradshteyn, I.S. and I. M. Ryzhik. 1980. Tables of integrals, series, and products. San Diego, CA, New York, etc., Acadcmic Press.
Laughton, A. S. 1983. Discussion comment. Philos. Trans. R. Soc. London, Ser. A, 309, 461 .

McGoogan, J. T. 1975. Satellite altimetry applications. IEEE Trans. Microwave Theory Tech., MTT-23, 970978.

Martin, T. V., H.J. Zwally, A.C. Brenner and R.A. Bindschadler. 1983. Analysis and retracking of continental ice sheet radar altimeter waveforms. 7. Geophys. Res., 88 C3), 1608-1616.

Papoulis, A. 1965. Probability, random variables and stochastic processes. New York, McGraw-Hill.

Proakis, J.G. and D. Manolakis. 1988. Introduction to digital signal processing. New York, Macmillan.

Remy, F., P. Mazzega, S. Houry, C. Brossier and J. F. Minster. 1989. Mapping of the topography of continental ice by inversion of satellite-altimeter data. J. Glaciol., 35(119), 98-107.

Walsh, E.J. 1982. Pulse-to-pulse correlation in satellite radar altimeters. Radio Sci., 17, 4.

Wingham, D.J. 1995. A method for determining the average height of a large topographic ice sheet from observations of the echo received by a satellite altimeter. 7. Glaciol., 41(137), 125-141.

Wingham, D.J., C. G. Rapley and J.G. Morley. 1993. Improved resolution ice sheet mapping with satellite radar altimeters. EOS, $\mathbf{7 4}(10), 113,116$.

MS received 15 September 1994 and accepted 30 Seplember 1994 